BULLETIN OF THE

AMERICAN MATHEMATICAL SOCIETY

Volume 79, Number 4, July 1973

\title{
GEODESICS OF SINGULAR RIEMANNIAN METRICS
}

\author{
BY ROBERT HERMANN
}

Communicated by François Trèves, December 18, 1972

1. Introduction. In this note, I shall briefly present a geometric notion, which I believe is new, and which may have interesting applications to physics (e.g. in the nonrelativistic limit of cosmological models) and to mathematics (e.g. the study of the boundary properties of the Bergmann metric on domains in complex Euclidean space). More details will appear in a forthcoming book [6].

What I shall do is to reformulate the notion of "geodesic" for a nonsingular Riemannian metric so that it makes sense in the singular case. This will require that the reader be familiar with the notion of the "Hamilton-Jacobi equation" associated with a variational problem. My book [1] can be used as a reference for this material and for the notations used in this note.

2. Geodesics of nonsingular Riemannian metrics. Let $M$ be a $C^{\infty}$ manifold. $V(M)$ denotes its $C^{\infty}$ vector fields, $F(M)$ its $C^{\infty}$, real-valued functions, and $F^{1}(M)$ its differential forms of degree one.

A Riemannian metric is usually defined as an $F(M)$-bilinear symmetric map $\beta: V(M) \times V(M) \rightarrow F(M)$ which is nondegenerate. Such a $\beta$ defines an $F(M)$-linear isomorphism $\alpha: F^{1}(M) \rightarrow V(M)$ with the following property:

$$
\alpha^{-1}(X)(Y)=\beta(X, Y) \text { for } X, Y \in V(M) .
$$

Let $\beta^{d}$ be the form: $F^{1}(M) \times F^{1}(M) \rightarrow F(M)$ defined as follows:

$$
\beta^{d}\left(\theta_{1}, \theta_{2}\right)=\beta\left(\alpha\left(\theta_{1}\right), \alpha\left(\theta_{2}\right)\right) \text { for } \theta_{1}, \theta_{2} \in F^{1}(M) .
$$

Definition. A function $f \in F(M)$ is a Hamilton-Jacobi function of the metric if there is a function $h($ ) of one variable such that:

$$
\beta^{d}(d f, d f)=h(f) \text {. }
$$

Let $\operatorname{grad}: F(M) \rightarrow V(M)$ be the first order linear differential operator defined as follows:

$$
\operatorname{grad} f=\alpha(d f) .
$$

THEOREM 2.1. A curve in $M$ is a geodesic of the nonsingular metric $\beta$ if and only if there exists, locally, a Hamilton-Jacobi function $f$ such that the curve is an integral curve of $\operatorname{grad} f$.

The proof of Theorem 2.1 is given in [1], and is a consequence of classical

AMS (MOS) subject classifications (1970). Primary 58C20.

Copyright (C) American Mathematical Society 1973 
Hamilton-Jacobi theory. This reformulation suggests the generalization of the "geodesic" notion to the singular case.

\section{Geodesics of singular metrics.}

Definition. A singular Riemannian metric is defined by an $F(M)$ bilinear, symmetric map $\gamma: F^{1}(M) \times F^{1}(M) \rightarrow F(M)$ which is degenerate, in the sense that there exists a point $p \in M$, and a form $\theta \in F^{1}(M)$ such that:

$$
\begin{array}{r}
\theta(p) \neq 0, \\
\gamma\left(\theta, F^{1}(M)\right)(p)=0 .
\end{array}
$$

Let $\gamma$ be such a singular metric. Let $\alpha$ be the $F(M)$-linear map $F^{1}(M) \rightarrow V(M)$ such that

$$
\theta_{1}(\alpha(\theta))=\gamma\left(\theta_{1}, \theta\right) \text { for } \theta, \theta_{1} \in F^{1}(M) .
$$

Let us say that a function $f \in F(M)$ is a Hamilton-Jacobi function if there is a function $h(\quad)$ of one variable such that:

$$
\gamma(d f, d f)=h(f) \text {. }
$$

Let us also say that a curve $\sigma$ is a geodesic of $\gamma$ if the following conditions are satisfied.

(a) $\sigma$ is $C^{\infty}$.

(b) $\sigma$ can be broken up into small pieces, in each of which it is an integral curve of a vector field of the form $\alpha(d f)$, where $f$ is a Hamilton-Jacobi function.

Definition. Let $p$ be a point of $M$. The metric component of $p$ is the set of all points $q \in M$ which can be joined to $p$ by a continuous, piecewise $C^{\infty}$ geodesic.

EXAMPLES. (A) $\gamma$ is identically zero. Then, every function is HamiltonJacobi, $\alpha(d f)=0$. The geodesics are points, and the metric components are also points.

(B) $M=R^{3}$, with coordinates $(x, y, z) \cdot \gamma$ is of the following form:

$$
\begin{aligned}
& \gamma(d x, d x)=0=\gamma(d x, d y)=\gamma(d x, d z)=\gamma(d y, d z), \\
& \gamma(d y, d y)=1=\gamma(d z, d z) .
\end{aligned}
$$

Then, $f(x, y, z)$ is Hamilton-Jacobi if and only if

$$
\left(\frac{\partial f}{\partial y}\right)^{2}+\left(\frac{\partial f}{\partial z}\right)^{2}=h(f), \quad \alpha(d f)=\frac{\partial f}{\partial y} \frac{\partial}{\partial y}+\frac{\partial f}{\partial z} \frac{\partial}{\partial z} .
$$

We see that the geodesics are curves $t \rightarrow(x(t), y(t), z(t))$ such that $x(t)=$ constant, $t \rightarrow(y(t), z(t))$ is a straight line of $R^{2}$. The metric component then forms a foliation of $M$, each diffeomorphic to $R^{2}$, with a 
nonsingular metric on each of the leaves.

4. Geodesics of singular metrics and foliations with singularities. Let $\gamma$ be a singular metric on $M$. Let $H$ denote the set of $\theta \in F^{1}(M)$ such that $\gamma\left(\theta, F^{1}(M)\right)=0$. Then, $H$ defines a Pfaffian system on $M$. Let $V$ denote the set of vector fields $X \in V(M)$ such that $\theta(X)=0$, for all $\theta \in H$. Then, $V$ defines a vector field system on $M$. We have $\alpha\left(F^{1}(M)\right) \subset V$. Thus, the geodesics are also integral curves of $V$. In determining the metric components, the results and idea from the theory of foliations-with-singularities [3], [4], [5] are obviously relevant. A recent definitive result in this theory by H. Sussmann [8] enables one to prove the following result, which was also pointed out to me by Sussmann:

THEOREM 4.1. The metric components are submanifolds of $M$.

5. Applications to the theory of second order differential operators. Let $N$ be a manifold, $\Delta: F(N) \rightarrow F(N)$ a $C^{\infty}$ second order differential operator on $N$. Let $O$ be an open subset of $N$, and let $M$ be a submanifold of $N$ that lies on the boundary of $O$. Suppose that $\Delta$ is tangent to $M$.

Often one encounters situations where the $\Delta$ is nonsingular on $O$, but "degenerates" on $M$. The symbol of $\Delta$ will be an $F(M)$-bilinear, symmetric map: $F^{1}(N) \times F^{1}(N) \rightarrow F(N)$. It will restrict to $O$ and $M$, hence will define a singular metric on $M$. The geodesics of this metric will play a key geometric role in the study of the analytic properties of $\Delta$ near the boundary. (See [2] for earlier work in this spirit.) For example, $N$ might be $C^{n}, O$ a bounded domain, $\Delta=$ Laplace-Beltrami-operator of the Bergmann metric on $O$. In several examples (e.g. $n=2, O=$ bidisk), the metric components, as defined above, coincide with the "boundary components", as defined by I. I. Piatetskii-Shapiro [7]. One may conjecture that this is true in some generality.

\section{BIBLIOGRAPHY}

1. R. Hermann, Differential geometry and the calculus of variations, Mathematics in Science and Engineering, Vol. 49. Academic Press, New York, 1968. MR 38 \# 1635.

2. - A Poisson kernel for homogeneous spaces, Proc. Amer. Math. Soc. 12 (1961), 892-899. MR 28 \# 4554.

3. - On the accessibility problem of control theory, Proceedings of the Symposium on differential equations, Colorado Springs, 1961, Localle and Lefschetz, (editors), Academic Press, New York.

4. The differential geometry of foliations. II, J. Math. Mech. 11 (1962), 303-315. MR 25 \# 5524.

5. - Cartan connections and the equivalence problem for geometric structures, Contributions to differential equations 3 (1964), 199-248. MR 29 \#2741.

6. - Geometric methods and ideas in physics and engineering (to appear).

7. I. I. Pjateckiri-Sapiro, Geometry of classical domains and the theory of automorphic function, Fizmatgiz, Moscow, 1961. MR 25 \#231.

8. H. Sussmann, Orbits of families of vector fields and integrability of distributions, Trans. Amer. Math. Soc., 180 (1973), 171-188. 08540

Department of Mathematics, Rutgers University, New Brunswick, New Jersey 